\title{
Construction of Distance Education Classroom in Architecture Specialty Based on Internet of Things Technology
}

\author{
http://dx.doi.org/10.3991/ijet.v11i05.5695 \\ Yuqiao Yang, Kanhua Yu \\ Chang'an University, Xi'an, Shanxi, China
}

\begin{abstract}
Internet of Things technology and industrial development will trigger a new round of information technology revolution and industrial revolution, and they are the commanding point of future competition in information industry and core driving force of industrial upgrade. This paper introduces current situation of distance teaching of Internet of Things and architecture specialties, designs and implements distance teaching experiment system platform for architecture specialty based on Internet of Things. This system is based on ZigBee /GPRS wireless network technology, sensor technology, embedded technology, Web distributed software technology and database technology. Besides, it adopts three interlinked networks and achieves efficient connection of multiple experiment terminals, servers and clients. As well, the information exchange is fast. Hence, it is convenient for practical application of distance teaching. The results of teaching experiment show that Internet of Things technology can improve students' academic performance and teachers' teaching effect. Therefore, it is a hot spot in modern teaching technology, so we should pay attention to it.
\end{abstract}

Index Terms-Internet of Things, distance teaching, Architectural Physics

\section{INTRODUCTION}

At present, Internet of Things which stretches across communication field and sensor field has become a research hotspot of various colleges and relevant enterprises [1]. "Internet of Things technology" refers to a network technology which extends and expands on the basis of internet technology. Its client extends and expands to any article for information exchange and communication. "Internet of Things technology" can connect any article with internet for information exchange and communication according to the agreed protocol through Radio Frequency Identification (RFID), infrared sensor, global positioning system, laser scanner and other information sensing devices so as to achieve intelligent identification, positioning, tracking, monitoring and management [2]. Internet of Things technology fully integrates embedded technology, sensor technology, RFID technology, wireless sensor network, IPv6 $\backslash$ ZigBee $\backslash$ GPRS $\backslash \mathrm{Wi}-\mathrm{Fi}$ and other communication network techniques. Therefore, it has wide application prospect in numerous fields.

Internet of Things is extensively applied in education, with significant advantages. For example, the voting device which is designed with active radio frequency can be used for statistics of students' voting and question answering, and the visual statistical graphic is displayed at the teacher side so that teachers can analyze the results and adjust teaching in time [3]. In special education, the sensor can be used to observe the attention of children with attention defect and collect movement signals so as to train the children with sensorimotor disorder and correct their movement [4]. Callaghan V et al. Callaghan V et al. did an experiment about the application of Internet of Things in education and discussed how to construct an intelligent teaching environment with Internet of Things. The results show that Internet of Things technology can enrich experiment-based teaching, assist teaching management and expands after-school education [5]. In experiment-based teaching, teachers and students can gather experimental data (such as temperature, humidity, angle and intensity of pressure) through remote control of experimental instruments after various sensors are installed on experimental instruments. The processed and analyzed results are provided for experimenters via wireless or wired network. Teachers and students can check and analyze data just through computer and other terminal devices. This guarantees comprehensiveness, authenticity and effectiveness of experimental data, achieves reform of experiment0based teaching mode, effectively enhances students' learning interest solves resource limitation in traditional classroom teaching and saves a large sum of funds [6].

As social economy and building industry develop rapidly, architecture specialty encounters a good development opportunity. To keep stable and sustainable development of building industry, there should be a large number of high-quality skill-oriented talents. They should not just own a wide range of theoretical knowledge and rich professional knowledge, but also have strong practical operation ability and skills, meet production and management demands of various building enterprises and possess integrated skills required by the grassroots posts. Meanwhile, they should have teamwork spirit as well as physical and psychological health and reach "zero-distance" post holding requirement [7]. At present, in terms of architecture teaching, traditional face-to-face teaching method is boring and difficult to stimulate learning initiative, and its limitation is significant [8,9]. Although the concept of distance education has been proposed for many years, it is short of strong platform design and system implementation. Distance education platform which is used by numerous colleges has such defects as single function, difficulty in sharing data and numerous limiting conditions. These give rise to serious convenience to teacher resource and teaching practice [10]. 
This research is devoted to using Internet of Things technology to develop a set of experiment system platform which can be used in architecture distance teaching in order to solve the problems of numerous experiment platforms, such as single function, strong independence, poor data shoring and many limiting conditions. This teaching experiment system platform owns multiple modes of information transmission ability and such advantages as rich functions, superior overall performance, remote manipulation and data sharing. Compared with other existing platform technology, this research adopts the design idea of combining soft with hardware, applies "three interlinked networks" for integrated design of multiple experimental facilities, information processing device and system control device and finally gets an experiment system platform with high performance and fast information exchange.

\section{Overall Scheme Of Distance Teaching}

\section{EXPERIMENT SYSTEM BASED ON INTERNET OF THINGS}

Distance teaching experiment system platform based on Internet of Things includes experiment terminal group which is composed of several experiment terminals, and each experiment terminal is designed as a node in Zig-Bee wireless network. Teaching server communicates with experiment terminal group through GPRS wireless network; student client communicates with teaching server through Internet wired network or GPRS wireless network; teacher client communicates with teaching server through Internet wired network or GPRS wireless network. Teacher client offers extra monitoring function as well as teaching content management and pouching functions. The detailed design block diagram is shown in Figure 1.

\section{A. Design of experiment terminal}

Each experiment terminal uniformly gathers various sensor data and carries out necessary protocol analysis and modeling analysis. When the analysis result exceeds the preset range, error will be reported. If necessary, relevant driver will be controlled for automatic emergence treat- ment. If emergency circumstances occur due to wrong operation or other reasons, the experiment terminal will automatically stop operation and inform the teaching server. After the teaching server stores the event to the log and send relevant information to the client, the client will pop up corresponding warning information. Then, students and relevant teachers can handle it in time to effectively avoid experiment accident.

\section{B. Design of teaching server}

Service software which is developed by Java language is deployed on the teaching server. In overall view of server bandwidth, data transmission mode and instantaneity, the software adopts B/S architectural pattern and object-oriented software design scheme, and the backend database uses MySQL. The software achieves GPRS wireless communication protocol at the teaching server side. The heartbeat polling mode is adopted to automatically allocate independent IP address for all experiment terminals and continuously receive measured data which are sent by each experiment terminal. After necessary redundancy removal, denoising and data fusion are conducted, the data will be stored in MySQL database as backup data. The teaching server software receives and handles all control operations send by student client and teacher client. To enhance control and avoid logic competition and potential conflict, priority-based transaction scheduling strategy algorithm is implemented. This strategy sets transaction priority send by the teacher client to the highest priority and priority processing right of server can be gained. The transaction priority send by the student client is set to a lower value, and the transaction may be interrupted and even cancelled by the transaction with high priority.

\section{Client design}

Client software can respond to user operation and send corresponding control operation request to the teaching server. After the treatment, the teaching server sends con-

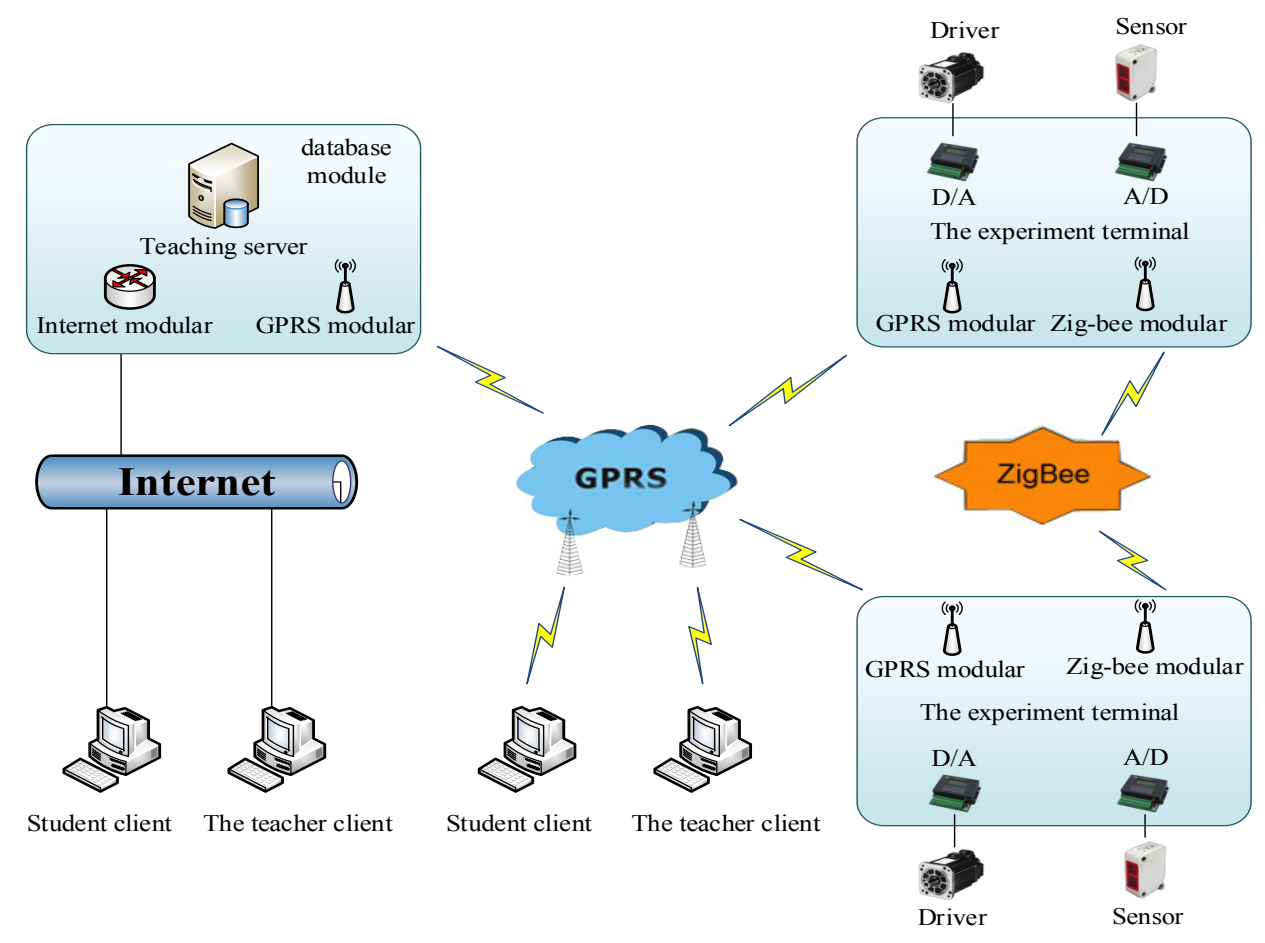

Figure 1. Hardware system structure 
PAPER

Construction of Distance Education Classroom in ARChitecture Specialty Based on InTERnet of Things...

trol order to each experiment terminal, and sends the images and data signals gathered by the sensor back to the client. The client displays the images of experiment terminal and relevant measured date to the interface in time so as to achieve experiment-based distance teaching function. Except distance experiment function, student client software also provides auxiliary functions including course selection, courseware learning, online examination and online question asking etc. This really achieves "learning anytime" in fragmented time, greatly improves interactive teaching experience and enhances students' learning interest. Compared with student client software, teacher client software also adds course maintenance, online question answering and other functions.

The detailed software process block diagram of distance teaching experiment system based on Internet of Things is shown in Figure 2. Main log-in interface of client software on laboratory PC is shown in Figure 3. Main operation interface of client software on laboratory $\mathrm{PC}$ is shown in Figure 4.

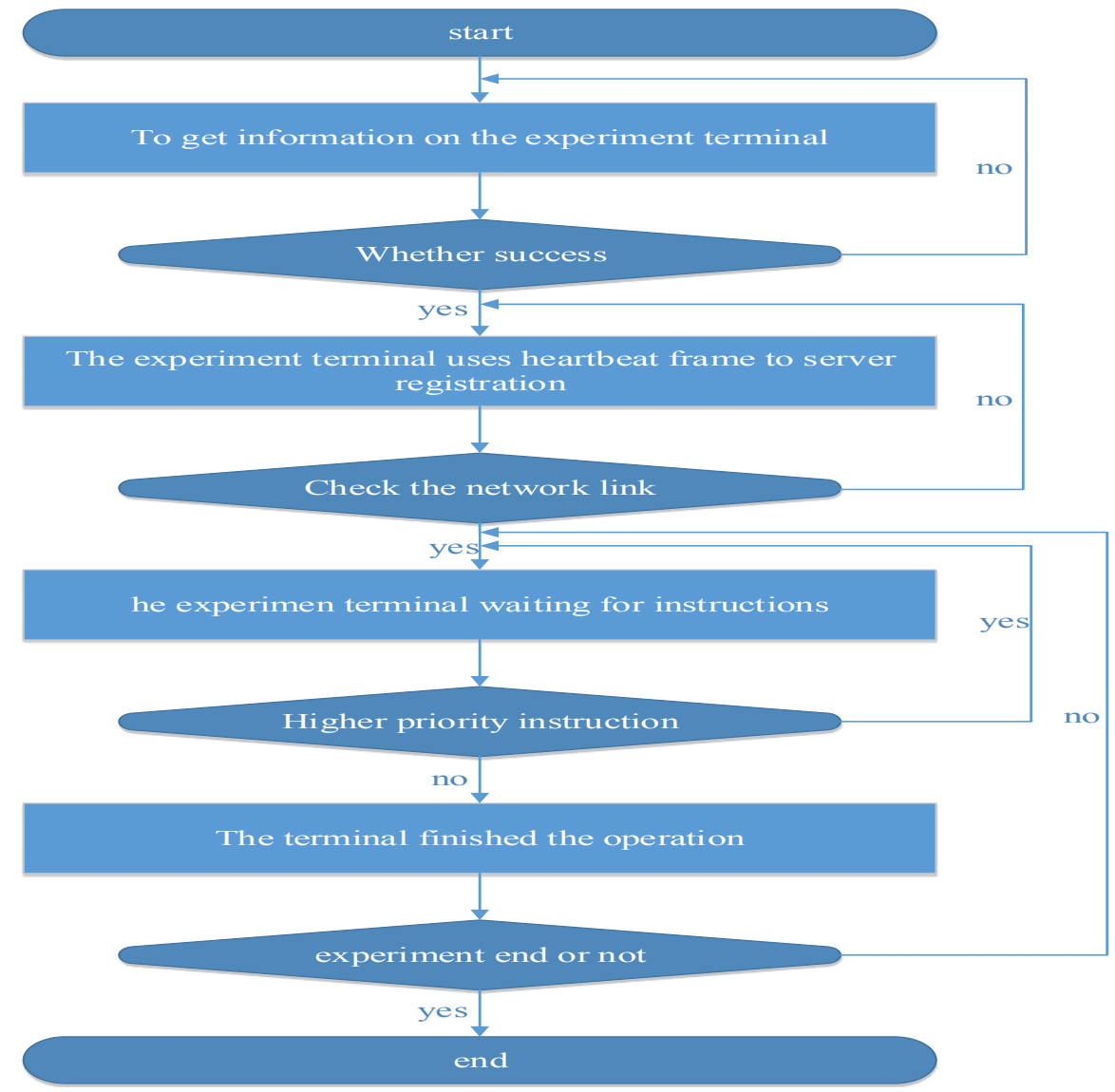

Figure 2. Software process block diagram

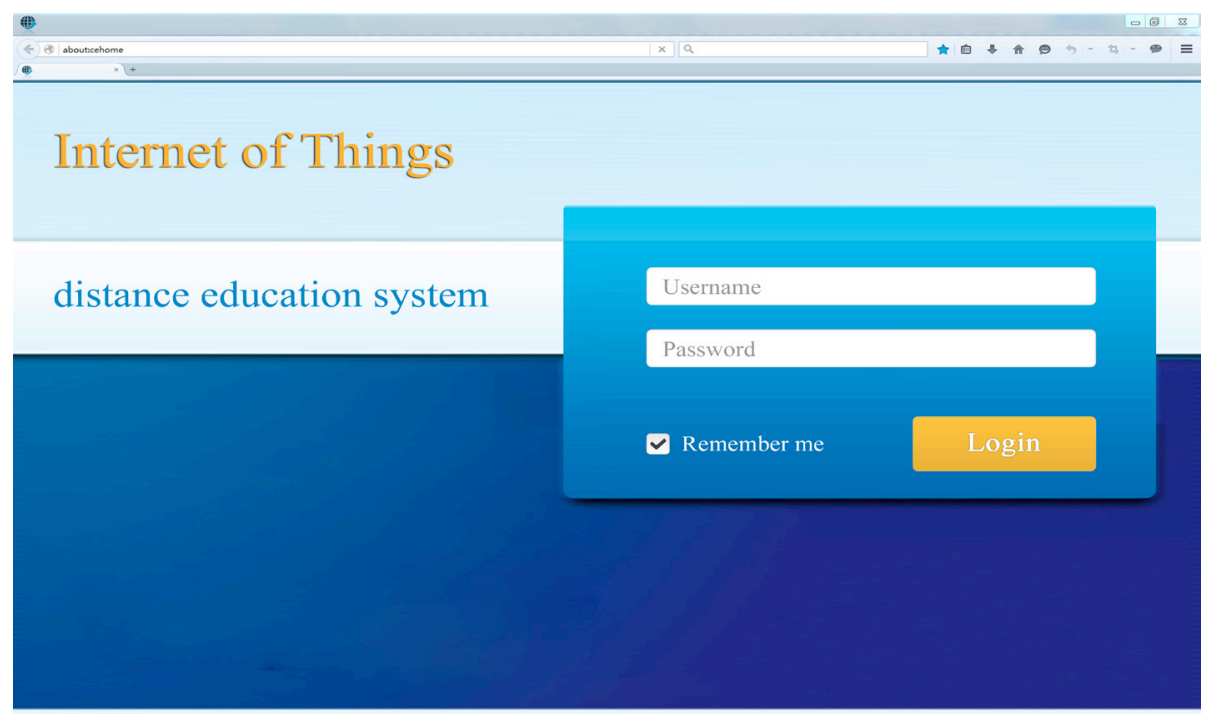

Figure 3. Main log-in interface of client software 
PAPER

Construction of Distance EduCAtion Classroom in ARChitecture Specialty Based on InTERnEt OF ThingS...

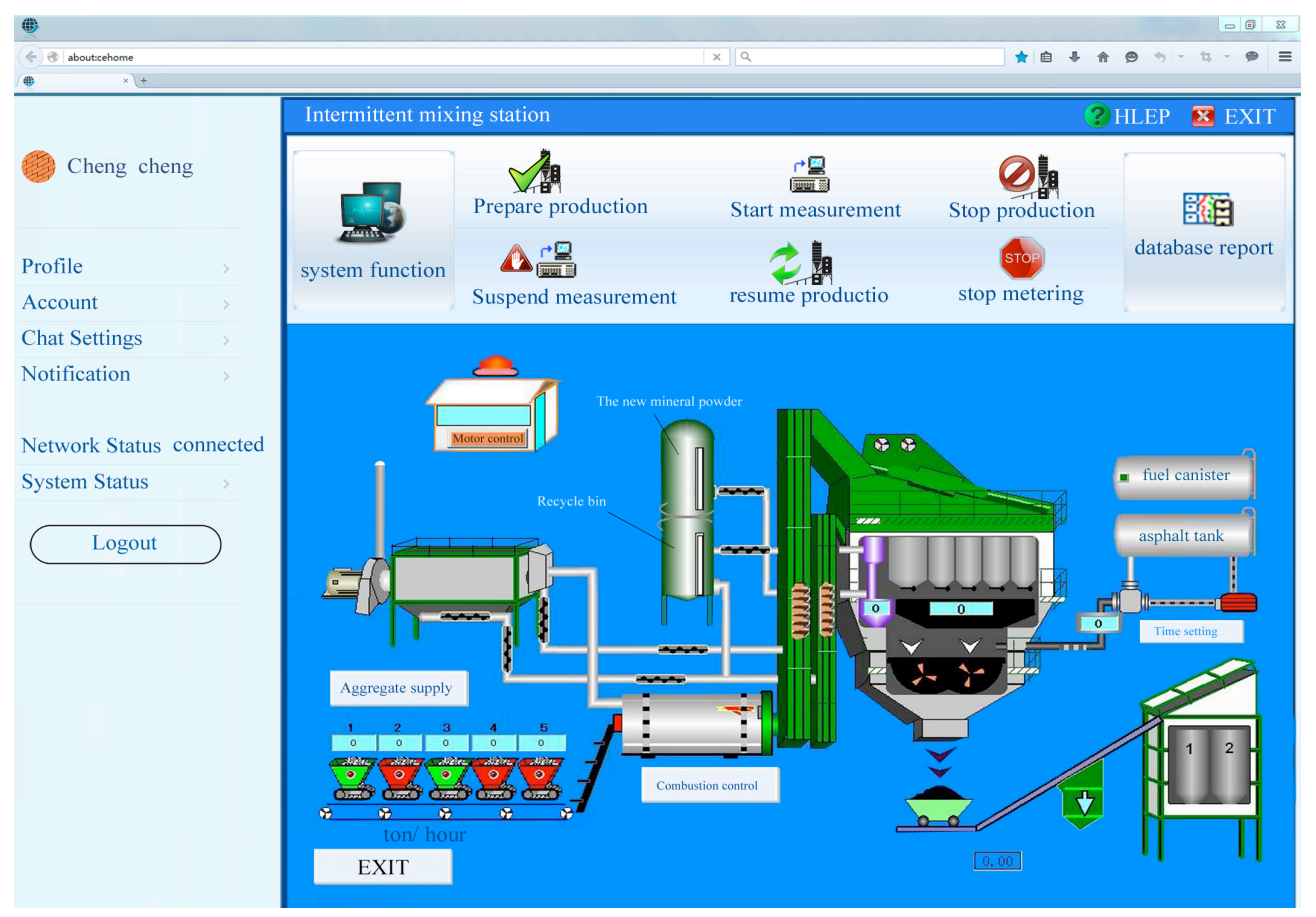

Figure 4. Experimental operation interface of client software

\section{PRACTICE IN ARCHITECTURE TEACHING}

\section{A. Course setting}

To investigate application effect of distance teaching experiment system platform based on Internet of Things and give full play to the advantage of Internet of Things technology, this research chooses a required course of architecture specialty in Chang'an University Architectural Physics experimental course as the content of teaching practice.

\section{B. Objects of experiment}

188 students majoring in architecture in College of Architecture and Urban Planning, Chang'an University who were admitted to the university in 2012 were chosen as the objects of research, including 99 male students and 89 female students. Their age ranged from 19 to 22, with the average age of 20.8 . They were grouped according to student number, and there were 10 groups in total. The group number was drawn at random. The control group included 95 students, and the experimental group included 93 students. The gender, age and college entrance examination achievement of students in both groups had no significant difference. Besides, the basic courses they learned were also basically consistent.

\section{Teaching method}

For the experimental group, distance teaching experiment system platform based on Internet of Things was adopted to research and develop experimental courseware of Architectural Physics. 8 innovative operation experiments whose difficulty increases gradually were designed by focusing on architectural thermal engineering, architectural optics and architectural acoustics of Architectural Physics. The experimental contents include Experiment I: indoor thermal and humid environment measurement experiment; Experiment II: outdoor thermal and humid environment measurement experiment; Experiment III: passive solar energy utilization design experiment; Exper- iment IV: architectural lighting calculation and design experiment; Experiment V: indoor environment lighting experiment; Experiment VI: indoor sound pressure calculation and measurement experiment; Experiment VII: indoor acoustic quality design experiment; Experiment VIII: building experiment for sound absorption and noise reduction.

The above 8 innovative experiments made students remotely control experiment terminal through the client. The driving device is operated remotely through Internet of Things technology, and data information of each sensor and image data on the sport are acquired. The client designs several experimental steps for each experiment and guides students to do the experiments by steps. After students complete all steps, the client will offer an open-type task testing in each experiment so that the students can carry out deeper exploration and research. All operation steps and experimental data are collected by the teaching server and stored in the database as important basis for final evaluation.

Traditional teaching method was adopted for the control group. The teacher taught experimental contents and steps before the experiments, and students finished various experiments of Architectural Physics in the laboratory. In case of any doubt, students might consult the teacher or group members.

\section{Teaching effect evaluation}

Teaching effect evaluation mainly involves two aspects: students' subjective evaluation of teaching effect; objective means is adopted to assess students' knowledge mastery degree.

After the teaching content for one semester was finished, the students in both groups took closed-book examination. The examination content included 25 one-choice questions ( 2 scores for each question), 10 true or false questions (1 score for each question) and 4 true or false questions (10 scores for each question). The full score is 100 scores. Meanwhile, Teaching Effect Questionnaire 
was distributed to all students. The questionnaire included 10 true or false questions, and students just needed to choose yes or no. 188 questionnaires were distributed in total, and 181 questionnaires were recovered, with the recovery rate of $96.3 \%$, including 181 effective questionnaires. The effective rate is $100 \%$.

\section{E. Statistical method}

SPSS V22 professional statistical software is applied in this paper for statistical analysis of teaching effects of students in both groups. Closed-book examination scores of students in both groups served as quantitative data, and the scores were processed into mean standard deviation for analysis. Independent-sample $T$ test was used for intergroup comparison. Students' subjective assessment of teaching effects served as qualitative data. The number of students who chose "yes" was processed into percentage for analysis. $\chi^{2}$ test was adopted inter-group comparison, with inspection level of $\alpha=0.05$.

\section{F. Result analysis and discussion}

(1) Comparison of students' knowledge mastery degree

Comparison of exam results of students in both groups is shown in Fig.5. Exam results of experimental group and control group are $86.5 \pm 7.2$ and $72.6 \pm 9.4$, respectively. It thus can be seen that exam result of experimental group is significantly higher than that of control group $(t=-13.27$, $\mathrm{P}<0.001)$. Hence, distance teaching system platform based on Internet of Things significantly improves students' scores.

(2) Comparison of students' subjective assessment of teaching effects
Table 1 shows students' subjective assessment of teaching effects. The evaluation was done from 8 aspects: deep impression on the content learned; able to understand the content learned; able to enhance operation ability; able to improve problem solving ability; able to enhance interest in the specialty; able to boost professional skills; able to improve self-study ability; able to promote comprehensive quality. The statistical results indicate that experimental group and control group have no significant difference in terms of teamwork ability and extracurricular knowledge expansion $(\mathrm{P}>0.1)$. In the other 6 aspects, the $\mathrm{P}>0.1$ is superior to the control group $(\mathrm{P}<0.1)$. Especially in the aspects of understanding the content learned, problem solving ability and self-study ability, the differences are very significant $(\mathrm{P}<0.01)$. Therefore, distance teaching experiment system based on Internet of Things technology can significantly improve teaching effect.

It can be seen that with the help of this system platform, students gain great help ad promotion in each link of teaching activity. In particular, distance teaching experiment system platform based on Internet of Things shows significant architecture teaching effect in such aspects as deep impression on the content learned, able to understand the content learned, able to enhance operation ability and able to improve self-study ability.

\section{CONCLUSIONS}

Distance teaching experiment system platform based on Internet of Things owns such advantages as rich functions, wide application scope, simple operation, strong system relevance and superior data communication and processing ability. It cannot merely promote distance teach-

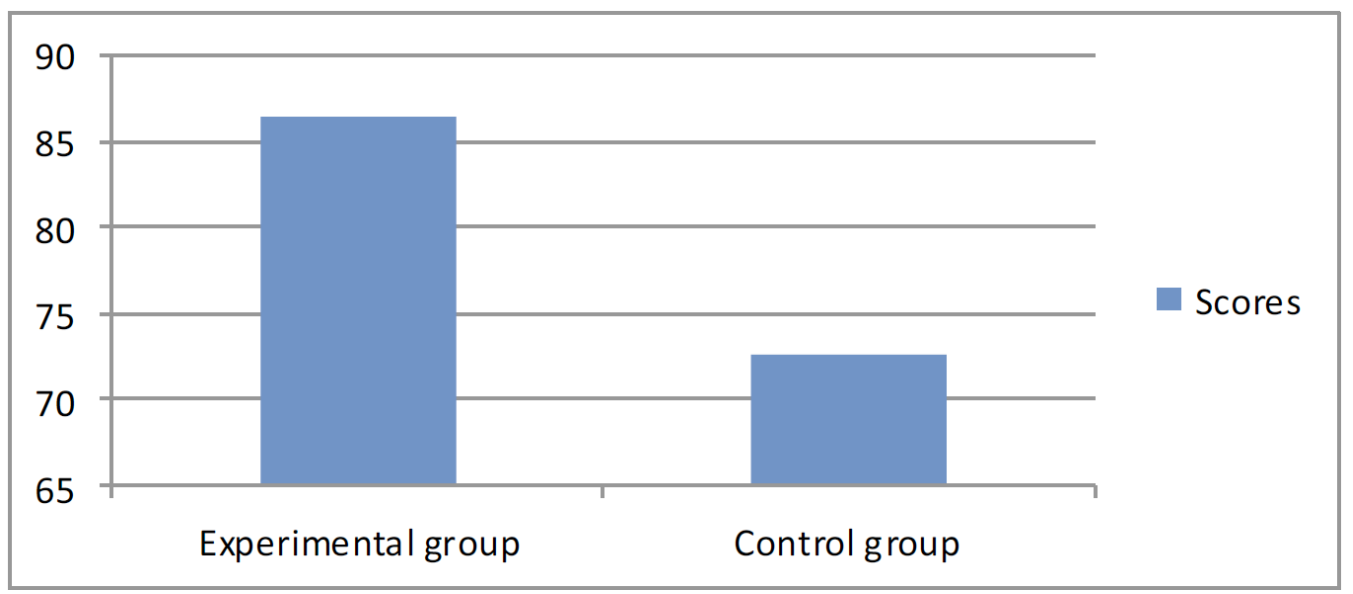

Figure 5. Comparison of exam results of students in both groups

TABLE I.

COMPARISON OF STUDENTS' ASSESSMENT OF TEACHING EFFECTS

\begin{tabular}{|c|c|c|c|c|c|}
\hline No. & Indicator & Experimental group(N=93) & Control group $(\mathrm{N}=95)$ & $\chi^{2}$ value & P value \\
\hline 01 & Deep impression on the content learned & $80(86.0)$ & $64(67.3)$ & 9.121 & 0.0025 \\
\hline 02 & Able to understand the content learned & $72(77.4)$ & $57(60.0)$ & 6.622 & 0.0101 \\
\hline 03 & Able to enhance operation ability & $81(87.0)$ & $70(73.6)$ & 5.348 & 0.0207 \\
\hline 04 & Able to improve problem solving ability & $78(83.8)$ & $64(67.3)$ & 6.925 & 0.0085 \\
\hline 05 & Able to improve teamwork ability & $30(32.2)$ & $29(30.5)$ & 0.065 & 0.7981 \\
\hline 06 & Able to enhance interest in the specialty & $76(81.7)$ & $66(69.4)$ & 3.814 & 0.0508 \\
\hline 07 & Able to boost professional skills & $69(74.1)$ & $59(62.1)$ & 3.160 & 0.0754 \\
\hline 08 & Able to improve self-study ability & $79(84.9)$ & $57(60.0)$ & 14.616 & 0.0001 \\
\hline 09 & Able to expand extracurricular knowledge & $43(46.2)$ & $44(46.3)$ & 0.0001 & 0.9913 \\
\hline 10 & Able to promote comprehensive quality & $70(75.2)$ & $60(63.1)$ & 3.231 & 0.0723 \\
\hline
\end{tabular}


ing efficiency and effect of architecture specialty greatly, but effectively facilitate students to more deeply learn and study theories and methods of architecture specialty. It has gained favorable teaching effect.

(1) Distance teaching experiment system platform based on Internet of Things significantly increases students' scores. When students learn with the help of distance teaching experiment system platform based on Internet of Things, they can acquire vivid and visual learning experience, which contributes to mastering and remembering the content learned. We have found that with such system platform, students gain great help and improvement in each link of teaching activity, especially in such aspects as deep impression on the content learned, able to understand the content learned, able to enhance operation ability and able to improve self-study ability. Thus, distance teaching experiment system platform based on Internet of Things has significant practical effect in architecture teaching, enhances students' learning enthusiasm and learning motivation and ultimately improves students' scores effectively

(2) Distance teaching experiment system platform based on Internet of Things significantly boosts course teaching effect. This teaching experiment system platform is further improved on the basis of original Internet of Things experiment platform. Since it owns multiple modes of information transmission ability, rich functions, superior overall performance, remote interaction operation and data resource sharing advantages, it relieves teachers' workload for lesson preparation to some degree. In addition, it contributes to integrating teaching resource ad superiority and boosts course teaching effect.

Moreover, we also have found in this teaching practice that, as the quantity of users rises fast, when students or teachers intensively $\log$ in the system in the middle term and the end of term, log-in failure situation will occur occasionally. When multiple experiment terminals work at the same time, massive measured data of each sensor will lose frame once in a while. This means this system still needs to be further improved and optimized during handling the business with high concurrency and high instantaneity. With rapid development of Internet of Things technology, distance teaching field based on such technology will certainly be applied in a deeper and wider way.

\section{REFERENCES}

[1] Sun Q.B., Liu J., Li S., Fan C.X., Sun J.J., "Internet of Things: Summarize on Concepts, Architecture and Key Technology Prob- lem," Journal of Beijing University of Posts and Telecommunications, vol. 3, no. 3, pp. 1-9, March 2010.

[2] Fu Q., Wei S.P., He L.X., "Study on Application of Wireless Sensor Network Education," China Educational Technology, no. 7, pp. 105-108, July 2008.

[3] Bandyopadhyay D., Sen J., "Internet of things: Applications and challenges in technology and standardization," Wireless Personal Communications, vol. 58, no. 1, pp. 49-69, April 2011. http://dx.doi.org/10.1007/s11277-011-0288-5

[4] Han Y.M., "Design of circuit simulation mechanism for deaf students," Experimental Technology and Management, vol. 28, no. 7, pp. 65-67, July 2011.

[5] Callaghan V., "Buzz-Boarding; practical support for teaching computing based on the internet-of-things," The Higher Education Academy-STEM, April 2012.

[6] He Z.Q., Zhuang J.M., “Application and Development Tendency of Internet of Things in Education," Modern Distance Education Research, no. 2, pp. 77-83, April 2011.

[7] Li F.H., Zhao Y.J., Meng F., "Teaching Reform of Civil Engineering Specialty Based on Excellent Engineer Education Program," Journal of Architectural Education in Institutions of Higher Learning, vol. 21, no. 6, pp. 40-42, December 2013.

[8] Ning B.K., Bao W.B., Huang J., \& Huang Z.Q., "On practice teaching of multivariate for civil engineering specialty and training application-oriented talent," Journal of Architectural Education in Institutions of Higher Learning, vol. 19, no. 1, 40 43, February 2010.

[9] Li S., Li J., "Skills and thinking about multimedia teaching of structural mechanics course for civil engineering specialty," Journal of Architectural Education in Institutions of Higher Learning, vol. 19, no. 1, 131 135, February 2010.

[10] Liu Z.Q., "Systematic research on graduation design teaching reform for civil engineering specialty," Journal of Architectural Education in Institutions of Higher Learning, vol. 19, no. 1, 118 121, February 2010 .

\section{AUTHORS}

Yuqiao YANG (corresponding author) is a Teacher of School of Architecture, Chang'an University, Xi'an 710061, Shanxi, China. His research interests include Internet of Things Technology and Micro-video Course. (yangyuqiao22@yeah.net)

Kanhua YU is an Associate profession of School of Architecture, Chang'an University, Xi'an 710061, Shanxi, China. His research interests include City Planning and Micro-video Course. (yukanhua@yeah.net)

This work was supported by National Natural Science Foundation of China (51378067), Social science fund of Shaanxi Province (213041140109) and Basic scientific research projects of Central University (310841155033). Submitted 29 March 2016. Published as resubmitted by the authors 05 May 2016. 